\title{
Interstitial Fluid in Lipedema and Control Skin
}

\author{
Marisol Allen, ${ }^{1}$ Michael Schwartz, ${ }^{2}$ and Karen L. Herbst ${ }^{1,3,4, *}$
}

\begin{abstract}
Background: Fluid in lymphedema tissue appears histologically as spaces around vessels and between dermal skin fibers. Lipedema is a painful disease of excess loose connective tissue (fat) in limbs, almost exclusively of women, that worsens by stage, increasing lymphedema risk. Many women with lipedema have hypermobile joints suggesting a connective tissue disorder that may affect vessel structure and compliance of tissue resulting in excess fluid entering the interstitial space. It is unclear if excess fluid is present in lipedema tissue. The purpose of this study is to determine if fluid accumulates around vessels and between skin fibers in the thigh tissue of women with lipedema. Methods: Skin biopsies from the thigh and abdomen from 30 controls and 80 women with lipedema were evaluated for dermal spaces and abnormal vessel phenotype (AVP): (1) rounded endothelial cells; (2) perivascular spaces; and (3) perivascular immune cell infiltrate. Women matched for body mass index (BMI) and age were considered controls if they did not have lipedema on clinical examination. Data were analyzed by analysis of variance (ANOVA) or unpaired $t$-tests using GraphPad Prism Software 7. $p<0.05$ was considered significant.

Results: Lipedema tissue mass increases beginning with Stage 1 up to Stage 3, with lipedema fat accumulating more on the limbs than the abdomen. AVP was higher in lipedema thigh ( $p=0.003)$ but not abdomen skin compared with controls. AVP was higher in thigh skin of women with Stage $1(p=0.001)$ and Stage $2(p=0.03)$ but not Stage 3 lipedema versus controls. AVP also was greater in the thigh skin of women with lipedema without obesity versus lipedema with obesity $(p<0.0001)$. Dermal space was increased in lipedema thigh $(p=0.0003)$ but not abdomen versus controls. Dermal spaces were also increased in women with lipedema Stage 3 $(p<0.0001)$ and Stage $2(p=0.0007)$ compared with controls.

Conclusion: Excess interstitial fluid in lipedema tissue may originate from dysfunctional blood vessels (microangiopathy). Increased compliance of connective tissue in higher stages of lipedema may allow fluid to disperse into the interstitial space, including between skin dermal fibers. Lipedema may be an early form of lymphedema. ClinicalTrials.gov: NCT02838277.
\end{abstract}

Keywords: lipedema; leaky lymphatics; interstitium; glycosaminoglycan; extracellular matrix; lymphedema; microangiopathy

\section{Introduction}

Lipedema is a painful disease of excess loose connective tissue (LCT: fat), almost exclusively in women, that resists loss by diet, exercise, or even bariatric surgery. ${ }^{1,2}$ The trunk is generally spared in lipedema resulting in a disproportionate accumulation of fat on the limbs. Lipedema begins or is exacerbated in periods of hor- monal flux when weight increases (puberty, pregnancy, and menopause), with surgery such as hysterectomy/ oophorectomy, and at other times of weight gain.

Lipedema is graded by stage: In Stage 1, the skin is smooth but the tissue under the skin has a pebble-like feel, which suggests fibrosis in the tissue. Lipedema Stage 2 is like Stage 1 except there is more lipedema tissue,

\footnotetext{
${ }^{1}$ Department of Medicine, TREAT Program, University of Arizona, Tucson, Arizona, USA.

${ }^{2}$ Pasadena Plastic Surgery, Pasadena, California, USA.

${ }^{3}$ Herbst Clinic, Tucson, Arizona, USA.

${ }^{4}$ Limitless Therapeutics and Karen L. Herbst MD, PC, Los Angeles, California, USA.

This study was presented at the International Lymphedema Framework meeting in Chicago, IL, June 2019.

*Address correspondence to: Limitless Therapeutics and Karen L. Herbst MD, PC, 12381 Wilshire Boulevard, Suite 202, Los Angeles, CA, 90025, USA, E-mail: kaherbst@gmail.com
}

(C) Marisol Allen et al., 2020; Published by Mary Ann Liebert, Inc. This Open Access article is distributed under the terms of the Creative Commons License (http://creativecommons.org/licenses/by/4.0), which permits unrestricted use, distribution, and reproduction in any medium, provided the original work is properly cited. 
the skin has dimpling due to fibrotic changes in the skin and underlying LCT, and nodules are larger. In Stage 3, there are large lobules of lipedema skin and tissue.

Lipedema tissue mass, BMI, and metabolic disease worsen by stage, ${ }^{3}$ and lymphedema is more common in Stage 3 compared with Stage 2 and Stage 1. Interestingly, women with lipedema can also have hypermobile joints, especially prominent in Stage $3 .^{5}$ Hypermobile joints suggest a connective tissue disorder such as hypermobile Ehlers Danlos spectrum disorder, ${ }^{6}$ associated with an increase in compliance and/or loss of elasticity to the tissue. In fact, people with the Williams-Beuren syndrome (OMIM 194050), who lack 1.5 million base pairs at 7q11.23 encompassing at least 17 genes, including the tropoelastin gene $(E L N)$, can also present with lipedema-like tissue. ${ }^{\text {? }}$

Connective tissue is integral to the structure of blood and lymphatic vessels, and therefore, genetic changes may affect vessel compliance resulting in an increase in permeability especially when under increased pressure. Al-Ghadban et al. showed that women with lipedema have increased numbers of microblood vessels in fat (angiogenesis) that are dilated and convoluted, suggesting a structural defect that could increase the risk for dysfunction and permeability. ${ }^{8}$ This publication also demonstrated that lipedema skin and fat had more blood than lymphatic vessels, suggesting a limited ability to remove excess fluid generated by higher microvessel permeability $^{8}$ in agreement with other published data. ${ }^{9}$

Excess fluid generated by microangiopathic blood vessels that is not picked up by lymphatic vessels would fill the interstitial space found throughout the body, ${ }^{10}$ resulting in enlargement of these areas. Vessels with greater permeability and increased size of interstitial spaces have been found in lymphedema, ${ }^{11}$ and capillaries surrounded by fluid suggesting hyperpermeability have been found in LCT of women with lipedema. ${ }^{12}$ Excess interstitial fluid may also make fat grow in lipedema as in lymphedema ${ }^{13,14}$ as lymph fluid is directly derived from interstitial fluid, which bathes cellular layers. ${ }^{15}$

Fluid shifts from the trunk to the lower body on standing increasing pressure in lower body arterial vessels, ${ }^{16}$ which could stress the structure of compliant vessels in lipedema, increasing interstitial fluid and fat growth on the thigh compared with the trunk. The aim of this study was to determine if an abnormal vessel phenotype (AVP) was present more often in the thighs compared with the abdomen in women with lipedema, in support of this hypothesis, and if blood vessels would have more AVP in women with lipedema compared with women without lipedema (controls). Spacing between dermal collagen fibers was used as a measure of increased interstitial fluid in the tissue.

\section{Methods}

Participants

All women were consented in writing before enrollment under a study approved by the University of Arizona Human Research and Protection Program. Women without lipedema were matched by age and BMI to women with lipedema as a comparator control population. Matching was accomplished with MedCalc Statistical Software (MedCalc Software Ltd, Ostend, Belgium). Women were considered to have obesity if their BMI was $\geq 30 \mathrm{~kg} / \mathrm{m}^{2}$.

\section{Biopsies}

Skin $5 \mathrm{~mm}$ punch biopsies were collected into zinc formalin from January 2017 to October 2019 from the thigh and/or abdomen of women with and without lipedema. Biopsies were processed into paraffin blocks, and then, slices cut and stained with hematoxylin and eosin (H\&E).

\section{Abnormal vessel phenotype}

All vessels in the papillary and reticular dermis of skin samples were scored for abnormal or normal blood vessel phenotype by microscopy at $40 \times$ magnification by two different observers. The number of abnormal vessels over the total number of vessels evaluated was multiplied by 100 to obtain a percent, and then, scores were averaged. A vessel was considered to have an abnormal phenotype if the following published criteria were met: (1) obvious perivascular space as in lymphedema $^{11,12}$; (2) rounded endothelial cells ${ }^{17,18}$; and (3) perivascular immune cell infiltrate (Fig. 1) ${ }^{8,19}$

\section{Interstitial space}

Five to eight images of the dermis at $40 \times$ magnification were obtained avoiding empty spaces beyond the skin biopsy. Dermal collagen was highlighted and quantitated as percent field (density) using Image,${ }^{20}$ and then averaged and subtracted from 100 to get percent dermal collagen space. Space was used as a surrogate measure of the amount of interstitial fluid between collagen fibrils.

\section{Statistics}

Data were analyzed by analysis of variance (ANOVA), linear regression, Pearson correlation, and unpaired 

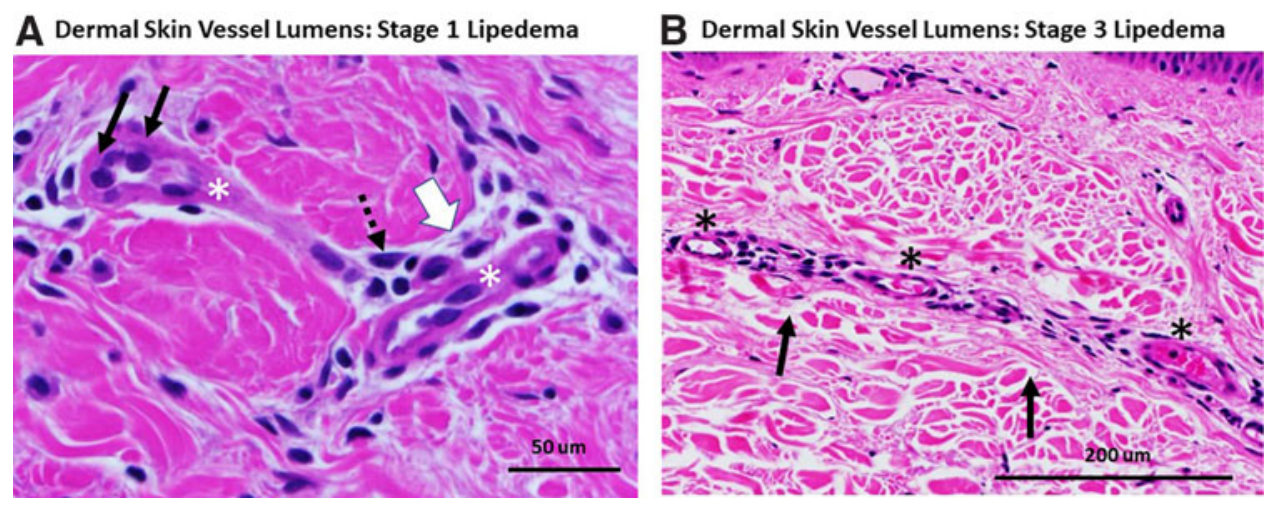

FIG. 1. Dermal vessels from thigh skin of women with lipedema. (A) Lumens of two dermal vessels $\left(^{*}\right)$ from a woman with Stage 1 lipedema. Abnormal vessel phenotype: black arrows denote rounded endothelial cells. Dotted arrow points to perivascular immune cell infiltrate. The white arrow denotes the enlarged perivascular space. Note the close packing of the maroon-colored collagen fibers in the dermis with almost no space between. H\&E. 60×. (B) An elongated vessel with multiple lumens $\left(^{*}\right)$ in the dermis of a woman with Stage 3 lipedema; the base of the epidermis can be seen at the top of the picture. Note the lack of AVP and the wide spacing of collagen fibrils in the dermis (black arrows). H\&E. $40 \times$ to allow clear visualization of spaces. AVP, abnormal vessel phenotype; $\mathrm{H} \& \mathrm{E}$, hematoxylin and eosin.

Student's $t$-tests as noted in the Figures (GraphPad Prism Software 7, San Diego, CA). Data are presented as mean \pm standard deviation. Significance was set at $p<0.05$.

\section{Results}

Demographics

Women with Stage 1 lipedema were matched with controls by age and BMI; women with Stage 2 lipedema were also matched with controls, noted in Figures. Women with Stage 3 lipedema were included in the study and compared with controls but not all were matched to controls as they tended to be older and have a higher BMI than available controls, consistent with lipedema as a progressive disease (Table 1).

\section{AVP in matched samples}

When examining skin punch biopsy samples of abdomen or thigh skin from women with and without lipedema matched to controls by BMI and age, women with lipedema had significantly more AVP in the thigh versus controls, but similar numbers of AVP in the abdomen compared with controls (Fig. 2).

\section{AVP in thigh skin in all stages of lipedema} versus controls

As women with Stage 3 lipedema have more lipedema tissue than earlier stages, it was hypothesized that they would have more AVP than women with Stage 1 or 2 lipedema. However, there were significantly

Table 1. Demographics of Women With and Without Lipedema

\begin{tabular}{|c|c|c|c|c|c|}
\hline \multirow[b]{2}{*}{ Demographics } & \multirow[b]{2}{*}{ Controls } & \multicolumn{3}{|c|}{ Lipedema stages } & \multirow[b]{2}{*}{ All lipedema participants } \\
\hline & & 1 & 2 & 3 & \\
\hline Number $(n)$ & 30 & 19 & 40 & 21 & 80 \\
\hline Age (years) & $38 \pm 13$ & $40 \pm 9$ & $49 \pm 11^{* * *}, \wedge$ & $51 \pm 10^{* * * \wedge}$ & $47 \pm 11$ \\
\hline $\mathrm{BMI}\left(\mathrm{kg} / \mathrm{m}^{2}\right)$ & $28.3 \pm 7$ & $26.3 \pm 3$ & $33.4 \pm 8^{*, \wedge \wedge}$ & $44 \pm 8^{* * *, \wedge \wedge \wedge,+}$ & $35 \pm 9$ \\
\hline White & 24 & 19 & 35 & 19 & 63 \\
\hline Non-Hispanic & 22 & 16 & 36 & 20 & 60 \\
\hline
\end{tabular}

Versus controls: ${ }^{*} p=0.01,{ }^{* *} p=0.0005,{ }^{* * *} p<0.0001$.

Versus L1: ${ }^{\wedge} p=0.01,{ }^{\wedge \wedge} p=0.002,{ }^{\wedge \wedge \wedge} p<0.0001$.

Versus L2: ${ }^{+} p<0.0001$.

BMI, body mass index; L1, lipedema Stage 1; L2, lipedema Stage 2. 

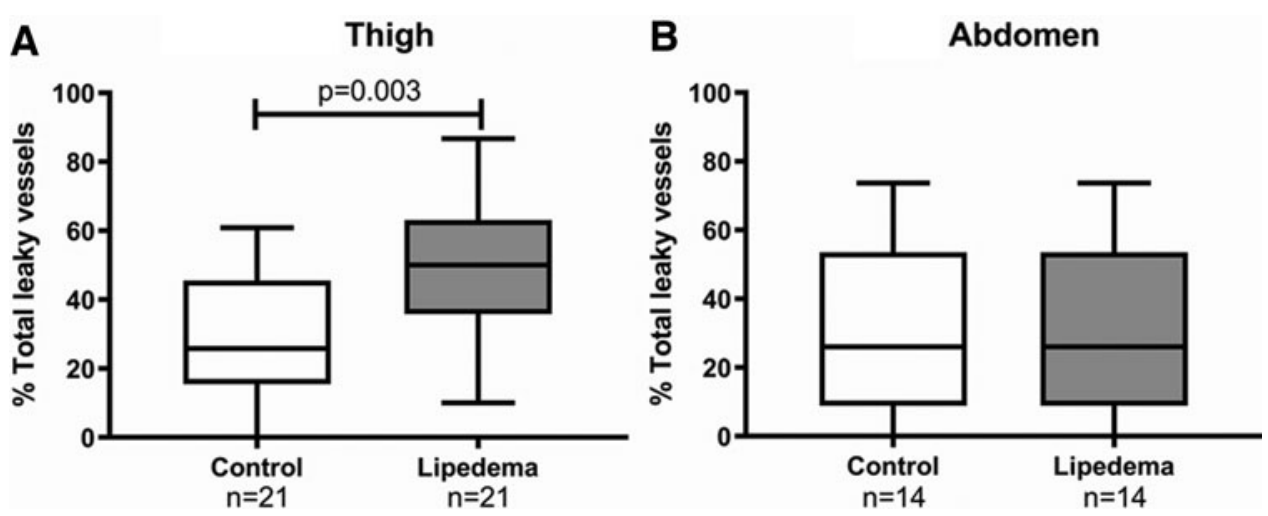

FIG. 2. Leaky vessel phenotype in women with and without lipedema (controls) matched for age and $\mathrm{BMI}$ for the thigh (A) and matched separately for age and BMI for the abdomen (B). Capped lines show significant differences between groups. Significance by unpaired $t$-test. BMl, body mass index.

more AVP in thigh skin of women with Stage 1 or 2 but not Stage 3 lipedema versus controls (Fig. 3). Simple linear regression showed a strong negative correlation of AVP with stage (Fig. 3).

\section{AVP and obesity}

Women with lipedema without obesity had more AVP in thigh skin compared with women with lipedema and obesity, or controls (Fig. 4). The greater the BMI, the fewer AVP in women with lipedema $\left(r^{2}=0.23 ; p<0.0001\right)$.

\section{Interstitial dermal spaces}

It was unclear why AVP were not as prominent in the thigh skin of women with Stage 3 lipedema. We hypothesized that increased compliance (change in interstitial fluid volume/change in interstitial pressure) in the tissue of women with Stage 3 lipedema allowed fluid to spread throughout the interstitial space. When examining abdomen and thigh dermal skin samples of women with and without lipedema, women with lipedema had significantly more space between collagen fibers in the dermis of the thigh $(46 \% \pm 3.5 \%)$ versus controls
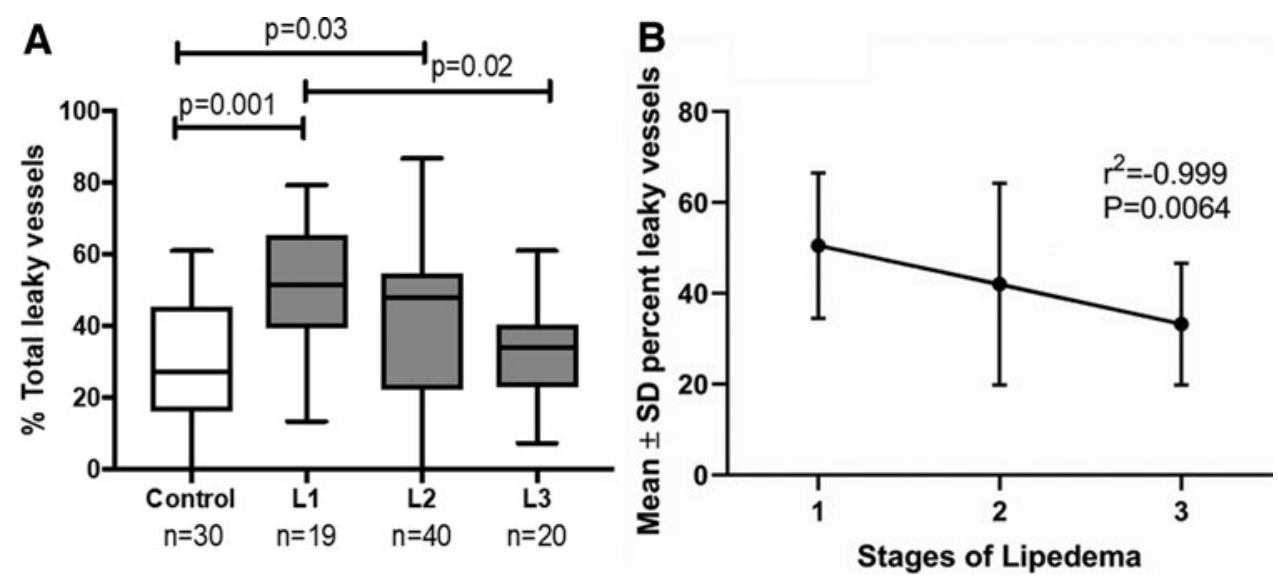

FIG. 3. Women with Stage 1 lipedema have a greater percentage of AVP in the thigh than women with Stage 3 lipedema or controls. (A) Percent vessels with AVP in controls and all stages of lipedema by ANOVA. Capped lines show significant differences between groups. (B) Simple linear regression of means of percent abnormal vessels from all stages of lipedema. L1, lipedema Stage 1; L2, lipedema Stage 2; L3, lipedema Stage 3. ANOVA, analysis of variance. 

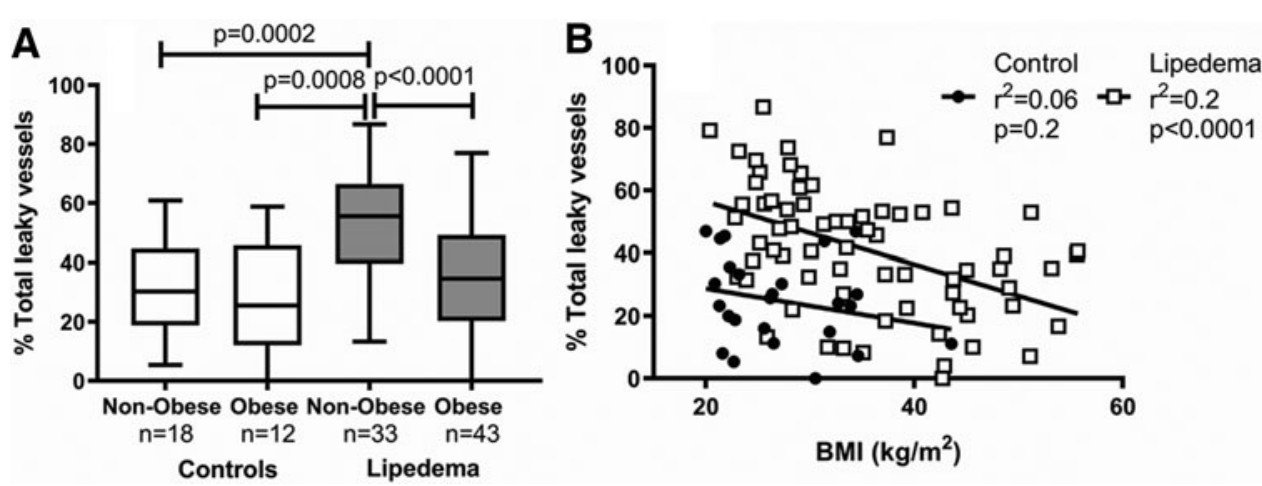

FIG. 4. (A) Women with lipedema who are not obese have more vessels with a leaky vessel phenotype in the thigh compared with women with lipedema with obesity, who are obese or controls with and without obesity by ANOVA. Capped lines show significant differences between groups. (B) There was a negative relationship between $\mathrm{BMI}$ and leaky vessel phenotype in women with lipedema but not controls by Pearson correlation.

$(42 \% \pm 2.9 \% ; p=0.003)$. Dermal spaces were similar on the abdomen $(42 \% \pm 5.4 \%)$ compared with controls $(43 \% \pm 2.6 \% ; p=0.7)$. Women with Stages 2 and 3 lipedema had greater space between collagen fibers in thigh skin than controls (Fig. 5). There was no correlation between space between collagen fibers and BMI in women with lipedema $\left(r^{2}=0.03 ; p=0.4\right)$ or controls $\left(r^{2}=0.15 ; p=0.08\right)$.

\section{Discussion}

Excess fluid that overwhelms lymphatic vessel function can result in lymphedema by the Starling rules for microvessels where lymphatic vessels supply the main exit route for fluid to leave tissue. ${ }^{21}$ Lipedema literally means fluid in the fat. In early stages, the fluid is nonpitting, so the fluid is dispersed in the tissue in the interstitial space and likely bound to glycosaminoglycans. Later,
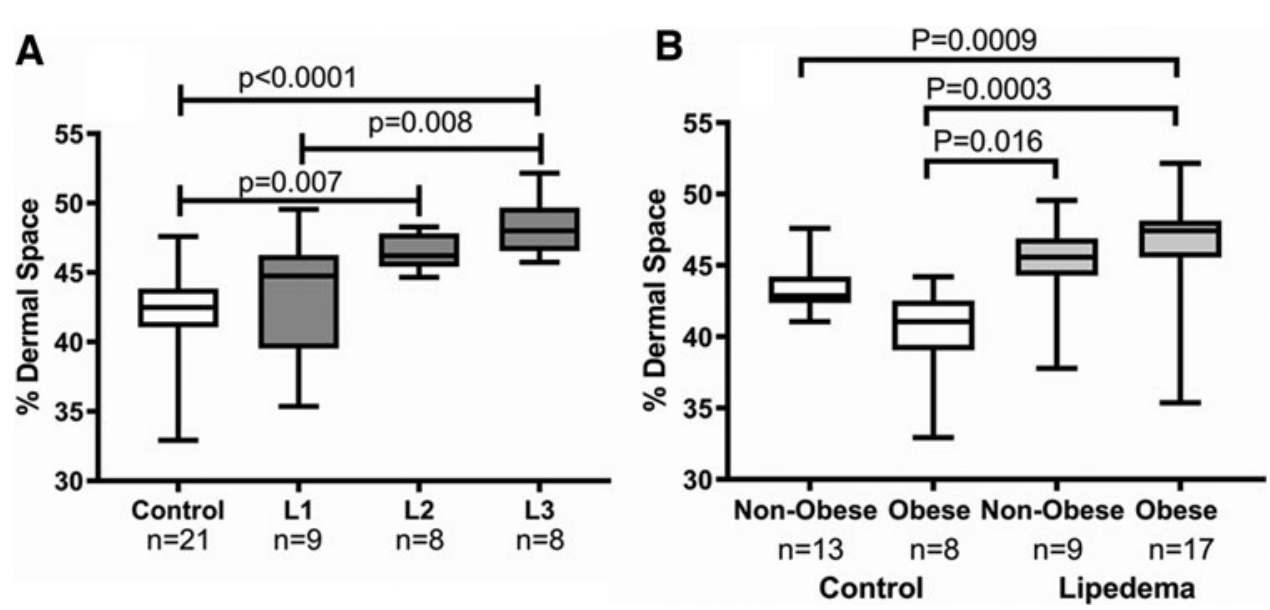

FIG. 5. Space between collagen fibers in the dermis of the thigh in controls and women with lipedema with or without obesity. (A) Collagen space was significantly greater in women with Stage 3 lipedema compared with women with Stage 1 lipedema or controls, and women with Stage 2 lipedema compared with controls by ANOVA. (B) Dermal spaces were significantly larger in thigh skin of women with lipedema whether obese or not obese compared with controls. Capped lines show significant differences between groups. L1, lipedema Stage 1; L2, lipedema Stage 2; L3, lipedema Stage 3. 
pitting edema can develop in lipedema similar to lymphedema. Blood vessel capillaries from lipedema tissue have been shown to be hyperpermeable, ${ }^{12}$ and thus could be one source of excess fluid entering the interstitial space. In support, there are increased blood vessels and microangiopathy in the skin and LCT of women with lipedema. ${ }^{8}$

We found AVP in the microvessels of the thigh but not abdomen of women with lipedema compared with controls. The thigh is an area of the body that has higher fluid column pressure when a person stands as fluid leaves the thorax. ${ }^{16}$ This pressure may be important in the development of lymphedema as disease onset is faster in the lower extremity after lymph node excision ${ }^{22}$ compared with the upper extremity. ${ }^{23}$ Excess lymph fluid makes fat grow, ${ }^{13,14}$ and therefore, it is not a stretch to consider that excess fluid in the tissue could also stimulate fat growth in lipedema.

The fact that so many women with lipedema have hypermobile joints suggests changes in connective tissue, including the structural connective tissue of vessels. Thus, higher hydrostatic pressure may pass more easily to the capillaries in the thighs of women with lipedema that then release excess fluid into the interstitial space. The abdomen as part of the trunk would not be as affected as the thigh due to lower fluid column pressure, consistent with our data of more AVP in the thigh versus the abdomen of women with lipedema.

Women with Stage 3 lipedema have more lipedema LCT. We hypothesized that women with Stage 3 lipedema would have a greater percentage of AVP in thigh skin vessels than women with Stage 1 lipedema. However, the opposite was found and in fact, the higher the stage, the lower the percent AVP in a highly statistically significant and linear manner. To better understand these data, we examined the interstitial space in the dermis. As in lymphedema, ${ }^{24}$ larger interstitial spaces were noted in the skin of women with Stage 3 compared with Stage 1 lipedema or controls. We hypothesize that dermal collagen may be more compliant in women with Stage 3 lipedema. Fluid that leaves vessels in the tissue of women with lipedema Stage 3 may enter the interstitial space and the poor elasticity (high compliance) of the tissue results in fluid stasis. This difference in compliance between Stage 1 versus Stage 3 tissue may reflect the contributions of different gene mutations. Mutations in genes that affect tissue compliance in the skin and vessels of women with Stage 3 lipedema may be different than mutations conferring compliance to microvessels of women with Stage 1 lipedema. For example, there are multiple reported genes that can confer a lipedema phenotype to tissue, and two of these genes affect tissue elasticity: ELN and TNXB (tenascin-X gene). ${ }^{25}$

The ability of dermal fibers to separate may be a marker of a hypermobile connective tissue disorder such as the hypermobile Ehlers Danlos hypermobile spectrum disorder. Our data also suggest that obesity alone does not drive the increased space between collagen in the dermis because women with lipedema with and without obesity had more space between collagen fibers in the dermis and greater AVP than controls with or without obesity. There are therefore inherent differences in the connective tissue between women with lipedema and controls, and obesity is not the driver for increased interstitial space or AVP.

Published data validate the findings of microangiopathy and angiogenesis in lipedema LCT. People with obesity (not lipedema) have lower adipose blood flow and calculated capillary diffusion capacity than leaner people ${ }^{26}$ whereas in lipedema, vessel density is higher ${ }^{8}$ and vessels more permeable as shown by our work here. Lymphatic vessels were smaller and less numerous in published data on women with Stage 3 lipedema. ${ }^{8}$ This suggests that both microangiopathy and lymphatic dysfunction play a role in fluid accumulation, more so in later stages of lipedema than in earlier stages where a microangiopathy may play a larger role initially. A mouse model of obesity with fewer numbers of lymphatic vessels ${ }^{27}$ supports this model, suggesting that obesity is important in lymphatic failure in later stage lipedema.

Higher limb capillary pressures, tissue structure changes, more blood vessels with excess permeability and poor lymphatic outflow all likely contribute to fluid accumulation in lipedema.

The microangiopathy in lipedema microvessels may be caused by disturbance of the glycocalyx that lines all vessels and is composed of proteoglycans, glycoproteins, and associated glycosaminoglycans. ${ }^{28}$ The tissue of women with lipedema has higher sodium concentrations. ${ }^{29}$ High sodium disturbs the glycocalyx barrier function of endothelial cells and predisposes the endothelium to inflammation. ${ }^{30}$ Low-sodium diets may improve the glycocalyx. ${ }^{31}$ More research is needed in this area.

If a microangiopathy and interstitial fluid bound to glycosaminoglycans are present early in lipedema tissue, treatment should aim at supporting the vessels in the legs by the use of the following: compression garments; hands on therapy to move fluid, sodium, and 
other prelymph components out of the tissue into lymphatic vessels; flavonoids and other anti-inflammatories from food and supplements to help reduce inflammation of the microvessels ${ }^{32}$; and possibly a low-salt diet to decrease glycosaminoglycan content. ${ }^{31}$

The data in this article were based on an AVP and collagen spacing that if incorrect as markers of leaky vessels and increased interstitial fluid, respectively, would negate our findings. However, our phenotype was based on published data demonstrating perivascular spaces around capillaries in fat tissue in lipedema, ${ }^{12}$ perivascular spaces ${ }^{11}$ and spaces between collagen fibers in the dermis ${ }^{24}$ in lymphedema, perivascular immune cell infiltrate in the skin in lipedema, ${ }^{8,19}$ and endothelial cell rounding as a marker of increased paracellular transport. ${ }^{17,18}$

\section{Conclusion}

Microangiopathy is likely present in women with early-stage lipedema that manifests more often in tissues exposed to higher hydrostatic pressure such as the thighs. In later stages, lymphatic dysfunction becomes an important contributor to tissue fluid. This excess fluid can reside around vessels or among tissue fibers. The increased fluid would account for the heaviness of the tissue as is often found in the legs of women with lipedema. Lipedema may be in the spectrum of lymphedema. The interstitial space and its glycosaminoglycans, the endothelial glycocalyx, and structure of the lipedema tissue need to be studied further.

\section{Acknowledgements}

We thank Anastasia Miramontes and Lyndsey Nermoe for their technical assistance.

\section{Author Disclosure Statement}

No competing financial interests exist.

\section{Funding Information}

This study was funded by the Lipedema Foundation.

\section{References}

1. Bast JH, Ahmed L, Engdahl R. Lipedema in patients after bariatric surgery. Surg Obes Relat Dis 2016;12:1131-1132.

2. Gensior MHL, Cornely M. Der Lipödemschmerz, seine Folgen auf die Lebensqualität betroffener Patientinnen-Ergebnisse einer Patientenbefragung mittels Schmerzfragebogen [Pain in lipoedema, fat in lipoedema and its consequences: Results of a patient survey based on a pain questionnaire]. Handchir Mikrochir Plast Chir 2019;51:249-254.
3. Torre YS, Wadeea R, Rosas V, Herbst KL. Lipedema: Friend and foe. Horm Mol Biol Clin Investig 2018;33: DOI: 10.1515/hmbci-2017-0076.

4. Herbst K, Mirkovskaya L, Bharhagava A, Chava Y, Te CH. Lipedema fat and signs and symptoms of illness, increase with advancing stage. Arch Med 2015;7:1-8.

5. Beltran K, Herbst KL. Differentiating lipedema and Dercum's disease. Int J Obes (Lond) 2017;41:240-245.

6. Tinkle B, Castori M, Berglund B, et al. Hypermobile Ehlers-Danlos syndrome (a.k.a. Ehlers-Danlos syndrome Type III and Ehlers-Danlos syndrome hypermobility type): Clinical description and natural history. Am J Med Genet C Semin Med Genet 2017;175:48-69.

7. Waxler JL, Guardino C, Feinn RS, Lee H, Pober BR, Stanley TL. Altered body composition, lipedema, and decreased bone density in individuals with Williams syndrome: A preliminary report. Eur J Med Genet 2017;60:250256.

8. AL-Ghadban S, Cromer W, Allen M, et al. Dilated blood and lymphatic microvessels, angiogenesis, increased macrophages, and adipocyte hypertrophy in lipedema thigh skin and fat tissue. J Obes 2019;2019: $1-10$.

9. Brautigam P, Foldi E, Schaiper I, Krause T, Vanscheidt W, Moser E. Analysis of lymphatic drainage in various forms of leg edema using two compartment lymphoscintigraphy. Lymphology 1998;31:43-55.

10. Benias PC, Wells RG, Sackey-Aboagye B, et al. Structure and distribution of an unrecognized interstitium in human tissues. Sci Rep 2018;8: 4947.

11. Olszewski WL, Ambujam PJ, Zaleska M, Cakala M. Where do lymph and tissue fluid accumulate in lymphedema of the lower limbs caused by obliteration of lymphatic collectors? Lymphology 2009;42:105111.

12. Curri SB, Merlen JF. [Microvascular disorders of adipose tissue]. J Mal Vasc 1986;11:303-309.

13. Schneider M, Conway EM, Carmeliet P. Lymph makes you fat. Nat Genet 2005;37:1023-1024.

14. Zampell JC, Aschen S, Weitman ES, et al. Regulation of adipogenesis by lymphatic fluid stasis: part I. Adipogenesis, fibrosis, and inflammation. Plast Reconstr Surg 2012;129:825-834.

15. Zawieja DC, Thangaswamy S, Wang W, et al. Lymphatic cannulation for lymph sampling and molecular delivery. J Immunol 2019;203:23392350

16. Hainsworth R. Arterial blood pressure. In: Henderby GEH, ed. Hypotensive anaesthesia. Edinburgh: Churchill Livingstone, 1985:3-29.

17. Cahill PA, Redmond EM. Vascular endothelium - gatekeeper of vessel health. Atherosclerosis 2016;248:97-109.

18. Malek AM, Izumo S. Mechanism of endothelial cell shape change and cytoskeletal remodeling in response to fluid shear stress. J Cell Sci 1996; 109:713-726.

19. Li CY, Kataru RP, Mehrara BJ. Histopathologic features of lymphedema: a molecular review. Int J Mol Sci 2020;21:E2546.

20. Schneider CA, Rasband WS, Eliceiri KW. NIH Image to ImageJ: 25 years of image analysis. Nat Methods 2012;9:671-675.

21. Levick JR, Michel CC. Microvascular fluid exchange and the revised Starling principle. Cardiovasc Res 2010;87:198-210.

22. Ryan M, Stainton MC, Slaytor EK, Jaconelli C, Watts S, Mackenzie $P$. Aetiology and prevalence of lower limb lymphoedema following treatment for gynaecological cancer. Aust N Z J Obstet Gynaecol 2003;43: 148-151.

23. Kwan ML, Darbinian J, Schmitz KH, et al. Risk factors for lymphedema in a prospective breast cancer survivorship study: The Pathways Study. Arch Surg 2010;145:1055-1063.

24. Olszewski WL, Zaleska M, Cakala M. Lymphedema is more than excess of fluid; a lympho-fibro-adipo-edema. Veins Lymphat 2018;7:7984.

25. Paolacci S, Precone V, Acquaviva F, et al. Genetics of lipedema: New perspectives on genetic research and molecular diagnoses. Eur Rev Med Pharmacol Sci 2019:23:5581-5594.

26. Coppack SW, Chinkes DL, Miles JM, Patterson BW, Klein S. A multicompartmental model of in vivo adipose tissue glycerol kinetics and capillary permeability in lean and obese humans. Diabetes 2005;54: 1934-1941.

27. Weitman ES, Aschen SZ, Farias-Eisner G, et al. Obesity impairs lymphatic fluid transport and dendritic cell migration to lymph nodes. PLoS One 2013;8:e70703. 
28. Tarbell JM, Simon SI, Curry FR. Mechanosensing at the vascular interface. Annu Rev Biomed Eng 2014;16:505-532.

29. Crescenzi R, Marton A, Donahue PMC, et al. Tissue sodium content is elevated in the skin and subcutaneous adipose tissue in women with lipedema. Obesity (Silver Spring) 2018;26:310-317.

30. Schierke F, Wyrwoll MJ, Wisdorf M, et al. Nanomechanics of the endothelial glycocalyx contribute to $\mathrm{Na}(+)$-induced vascular inflammation. Sci Rep 2017;7:46476.

31. Sugar D, Agocs R, Tatar E, et al. The contribution of skin glycosaminoglycans to the regulation of sodium homeostasis in rats. Physiol Res 2018; 67:777-785.

32. Zaragoza C, Villaescusa L, Monserrat J, Zaragoza F, Alvarez-Mon M. Potential therapeutic anti-inflammatory and immunomodulatory effects of dihydroflavones, flavones, and flavonols. Molecules 2020;25: 1017.
Cite this article as: Allen M, Schwartz M, Herbst KL (2020) Interstitial fluid in lipedema and control skin, Women's Health Reports 1:1, 480487, DOI: $10.1089 /$ whr.2020.0086.

\section{Abbreviations Used}

ANOVA $=$ analysis of variance

AVP $=$ abnormal vessel phenotype

$\mathrm{BMI}=$ body mass index

$\mathrm{H} \& \mathrm{E}=$ hematoxylin and eosin

$\mathrm{LCT}=$ loose connective tissue
Publish in Women's Health Reports

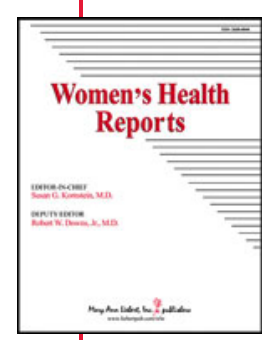

- Immediate, unrestricted online access

- Rigorous peer review

- Compliance with open access mandates

- Authors retain copyright

- Highly indexed

- Targeted email marketing

liebertpub.com/whr 